\title{
\begin{tabular}{|c|l|}
\hline IV Simpósio Paranaense de Modelagem, & Artigo: \\
$\begin{array}{c}\text { Simulaçãoe Controle de Processos } \\
\text { ISSN : 1984-7521 }\end{array}$ & Páginas:241 - 245
\end{tabular}
}

\section{ALGORITMOS HEURÍSTICOS PARA SINTONIA DE CONTROLADOR PID}

\author{
Felipe Tozim Demiti ${ }^{1^{*}}$, Cid Marcos G. Andrade ${ }^{2}$ \\ 1 - Universidade Estadual de Maringá - UEM - PR, felipetozim2@ gmail.com \\ 2 - Universidade Estadual de Maringá - UEM - PR, Maringá - PR
}

Resumo - Este trabalho tem o objetivo de propor dois métodos alternativos de sintonia de controladores PID, em relação ao método de Ziegler-Nichols. Métodos estes que são baseados em meta-heurísticas (algoritmos genéticos e evolução diferencial). Inicialmente é feita uma breve introdução sobre a sintonia de controladores. Após, são apresentados o Scilab (programa computacional usado para a simulação) bem como os algoritmos de sintonia baseados nas meta-heurísticas. Depois, são apresentados e discutidos os resultados obtidos com as simulações, que aliás comprovam que o método tradicional de sintonia de Ziegler-Nichols pode ser superado com o uso das meta-heurísticas já mencionadas. Ao fim conclui-se que a qualidade das respostas da sintonia via $\mathrm{AG}$ e via DE foi melhor, graficamente e em termos de ISE, quea sintonia via Ziegler-Nichols.

Palavras-chave: Algoritmos meta-heurísticos; Sintonia; Controlador PID; Algoritmo Genético; Evolução Diferencial.

\section{INTRODUÇÃO}

A importância de controladores que fazem uso das ações Proporcional, Integral e Derivativa (PID) é muito clara, principalmente em processos industriais, em que cerca de $95 \%$ das malhas de controle utilizadas possuem este tipo de controlador [1]. Fato este que contribui para o controlador PID ser considerado uma opção de solução confiável, poderosa e de fácil implementação [1].

Para que um controlador PID desempenhe de modo adequado sua função, este deve ser ajustado ou sintonizado. A sintonia de um PID pode ser definida, em resumo, como a busca pelos valores mais adequados dos ganhos das respectivas ações. Os ganhos proporcional $\left(K_{p}\right)$, integral $\left(K_{i}\right)$ e derivativo $\left(K_{d}\right)$ compõem a Função de Transferência (FT) do PID, que é representada no domínio da frequência pela Eq. 1.

$$
G_{c}(s)=K_{p}+\frac{K_{i}}{s}+K_{d} s
$$

Ao longo dos anos, diversas técnicas de sintonia já foram propostas a fim de que o ajuste do PID fosse facilitado e ao mesmo tempo conseguisse fornecer um controle que atendesse aos critérios de desempenho do sistema controlado. Entre os métodos determinísticos, o de Ziegler-Nichols é um dos mais tradicionais. Este trabalho tem o objetivo de propor técnicas de sintonia baseadas em algoritmos meta-heurísticos (Algoritmo Genético (AG), Evolutivo Diferencial (ED). O intuito é demonstrar, através de simulação computacional, que tais métodos podem ser superiores, em termos de qualidade de sintonia, em relação ao método de $\mathrm{ZN}$.

\section{MATERIAIS E MÉTODOS}

Para o desenvolvimento deste trabalho os recursos (materiais) usados foram um computador do tipo notebook e o software Scilab. Com relação aos métodos, optou-se pela sintonia do PID segundo o método de $\mathrm{ZN}$ e com as técnicas AG e DE, com algumas modificações em relação a versão original desses métodos meta-heurísticos.

\section{4 e 15 de março de 2019 Curitiba - Paraná}


Para a simulação, considerou-se a FT de um sistema conectado em cascata a FT de um PID, com este subsistema apresentando uma realimentação negativa (feedback) como ilustrado pela Fig. 1.

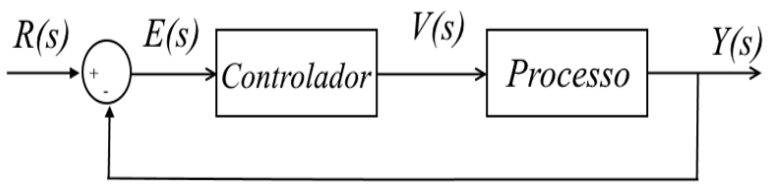

Figura 1 - Sistema de referência para os algoritmos de sintonia.

O principal aspecto da simulação é a análise da resposta a uma entrada em degrau unitário ao sistema representado pela Fig. 1. Para tal, foi empregado o critério de desempenho baseado na integral do erro quadrático (Integrated Square Error - ISE) somado ao sobressinal. $O$ foco das simulações era reduzir o valor dessa soma em relação ao PID sintonizado por ZN, com o uso das meta-heurísticas já citadas.

\section{SCILAB}

O programa escolhido para as simulações foi o Scilab. Ele foi instalado em um computador com sistema operacional Windows 10 Home Single Language (64 bits). O hardware dessa máquina é formado por um processador Intel ${ }^{\circledR}$ Core $^{\mathrm{TM}}$ i5-7200U de sétima geração e velocidade entre 2,50 e 2,70 GHz, HD de 1 TB e memória RAM de 8 GB.

Este programa é um software livre com código aberto, que pode ser utilizado para aplicações científicas e de engenharia, principalmente em problemas de computação numérica [2]. As características fundamentais desse programa são [2]: linguagem de programação de alto nível, uma grande quantidade de funções matemáticas; funções gráficas em duas e três dimensões; capacidade de trabalhar com polinômios e matrizes.

\section{ALGORITMOS DE SINTONIA}

Resumidamente, nesta parte deste trabalho, os algoritmos de sintonia AG e DE serão explicados e as principais modificações serão destacadas. Vale ressaltar que, todos os algoritmos de sintonia foram construídos na linguagem do Scilab e que todos possuem a mesma função objetivo (função de avaliação) que tem como parâmetro de avaliação a soma da ISE com o sobressinal da resposta em questão.

Com relação ao AG, a primeira etapa consiste na inicialização de parâmetros. Especificamente no tamanho da população, número máximo de iterações, tamanho do espaço de busca, probabilidades de cruzamento e de mutação bem como o número de indivíduos escolhidos no processo de seleção. Na sequência, de modo aleatório é gerada uma população inicial de soluções e estas são avaliadas pela função objetivo. A criação da população inicial é feita por meio da diferença entre metade do limite superior do espaço de busca com um terço do limite inferior do intervalo de busca, multiplicado por um número gerado aleatoriamente. Ao resultado dessa operação é somado um terço do limite inferior do espaço de busca.

Após, o laço de repetição é iniciado e só é encerrado quando o número máximo de gerações é atingido. Dentro desse laço, o primeiro operador a atuar é o de cruzamento, em que dois indivíduos têm suas informações combinadas por meio de operações aritméticas. Nem todos os indivíduos sofrem cruzamento, isso vai depender da taxa de cruzamento escolhida. O próximo operador é o de mutação. Ele tem um diferencial em relação ao que existe nos AGs mais comuns, em que um vetor com valores gerados aleatoriamente (com distribuição gaussiana), tem seus valores adicionados aos valores da população que sofreu o cruzamento. No algoritmo empregado neste trabalho, 
observou-se que o uso de uma distribuição qui quadrado (com grau de liberdade igual ao tamanho da população) e a subtração ao invés da adição, proporciona uma melhor performance do AG.

Ainda dentro do laço de repetição, a nova população é novamente avaliada. Após, o operador seleção (que faz uso do método torneio) é executado. Ele seleciona de modo randômico uma quantidade de indivíduos preestabelecida. O indivíduo com melhor avaliação dentre os selecionados irá compor a próxima geração.

$\mathrm{Na}$ sequência, são aplicadas práticas de elitismo, em que o melhor indivíduo da população atual é duplicado e substitui o pior indivíduo. Esta é a última instrução executada dentro do laço de repetição. Por fim, quando o laço se encerra, a melhor solução é exibida.

Para o algoritmo de sintonia via $\mathrm{DE}$, primeiramente são inicializados os parâmetros taxa de cruzamento, fator de escala, limites do espaço de busca e a quantidade máxima de iterações. Após, aleatoriamente é gerada uma população de soluções iniciais. O que acontece através do seguinte procedimento, o usuário do algoritmo fornece dois valores distintos, então determina-se a diferença entre esses valores e ao resultado disto é multiplicado um valor gerado aleatoriamente. Ao resultado destas operações é adicionado o menor valor fornecido pelo usuário, o que encerra o processo de produção da população inicial de soluções. Elas são então avaliadas pela função objetivo a fim de se determinar o melhor indivíduo desta geração, que servirá de comparativo inicial para determinar a melhor solução global.

Na sequência, tem início o laço de repetição, cujo critério de parada é o número máximo de iterações definido anteriormente. Dentro dele, inicialmente é executado o operador mutação, que apresenta uma modificação em relação aos algoritmos DE mais tradicionais. Nesse caso, dois indivíduos são escolhidos aleatoriamente, é feita a diferença entre eles e ao resultado dessa operação é multiplicada a constante fator de escala, bem como um número gerado aleatoriamente. Ao resultado de todas estas operações é adicionado o melhor indivíduo da geração anterior, o que finaliza o processo de mutação.

O próximo operador executado é o de cruzamento. Nele, segundo a taxa de cruzamento estabelecida, são escolhidos indivíduos da geração anterior e da atual para serem combinados (na prática eles são somados). Após, é empregado o operador seleção, que compara as soluções da geração anterior e da atual. Caso haja uma solução melhor no primeiro, ela substitui a solução que ocupa o mesmo índice de vetor no segundo caso (geração atual). Por fim, quando o laço de repetição é encerrado, a melhor solução é apresentada.

\section{CONFIGURAÇÕES, RESULTADOS E DISCUSSÕES}

O sistema escolhido para ser controlado pelo PID tem uma FT representada pela Eq. 2, ela foi modificada de [3] e modela um dispositivo experimental para controle de temperatura.

$$
\frac{0,1312}{315 \cdot s+1}
$$

Através de tentativa e erro os parâmetros de cada algoritmo foram configurados. Tanto o AG quanto o DE tiveram os mesmos valores escolhidos, no caso do número máximo de iterações (50), limite do espaço de busca (0 a 10000 para os três ganhos) e intervalo de tempo para o cálculo da resposta ao degrau (0 a 100 s). Em relação ao AG, optou-se por uma população com 15 indivíduos, probabilidade de cruzamento de 0,8 e probabilidade de mutação de 0,9 e 3 indivíduos para o processo de seleção. No que diz 
respeito ao DE a população tem um tamanho de 30 indivíduos, taxa de cruzamento de 0,9 e fator de escala de 0,8 .

\section{RESULTADOS E DISCUSSÃO}

Para a sintonia via $\mathrm{ZN}$, como este sistema é desprovido de atraso, as regras desse método indicam que apenas uma ação proporcional com elevado valor é necessária, devendo a ação integral e a derivativa serem iguais a zero. Através de testes, constatouse que uma sintonia com aproximadamente $K_{p} \geq 1250, K_{i}=K d=0$, tem o critério de desempenho ISE com um valor inferior a um. Então, estes foram os valores escolhidos para a sintonia via ZN. Eles produziram uma sintonia com uma ISE = 0,96968, como registrado na Tab. 1.

Tabela 1 - Ganhos para ajuste do PID e critérios de desempenho para cada método de sintonia

Ganho Critérios de desempenho

\begin{tabular}{ccccc} 
Método & $\mathrm{K}_{\mathrm{p}}$ & $\mathrm{K}_{\mathrm{i}}$ & $\mathrm{K}_{\mathrm{d}}$ & ISE \\
& & & 0 & 0.96968 \\
ZN & 1250.0000 & 0 & 0 & 0.08318 \\
$\mathrm{AG}$ & 4755.9132 & 0.3335 & 4827.7126 & 0.22299 \\
$\mathrm{DE}$ & 2984.1522 & 2.3792 & 1960.9124 & \\
\hline
\end{tabular}

Graficamente, a sintonia da Eq. 3 via ZN está representada na Fig. 2. Nela, bem como nos demais gráficos é apresentada uma ampliação do gráfico original em virtude da manutenção da conformidade da resposta.

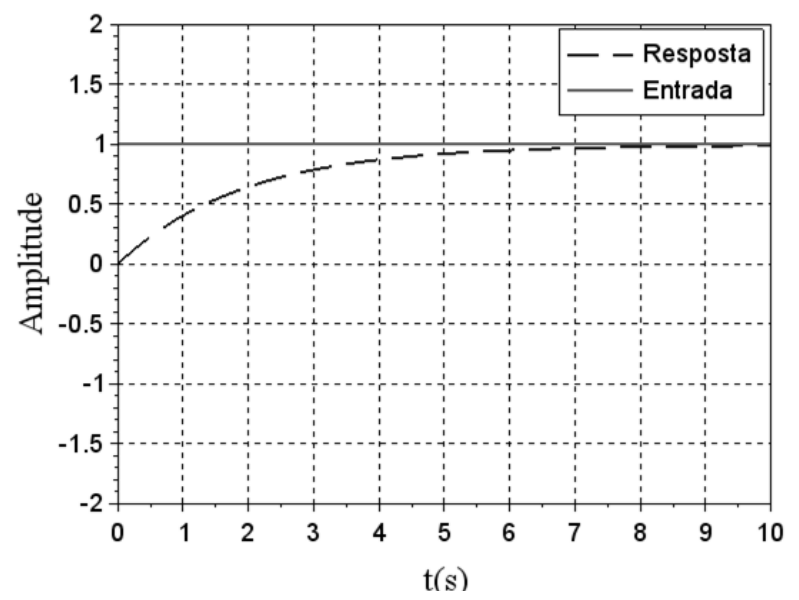

Figura 2 - Resposta do sistema da Eq. 3 sintonizado via ZN.

Para a determinação da melhor sintonia por AG e DE, cada método foi executado cinco vezes e a sintonia que forneceu o menor ISE, para cada método, foi escolhida como a melhor e incluída na Tab. 1. Por meio dos dados dessa tabela é possível verificar que a sintonia via $\mathrm{ZN}$ foi a que obteve o pior valor da ISE, sendo superado pelas sintonias por AG (ISE = 0,08318) e DE (ISE = 0,22299). O que indica que o método clássico de ZN pode sim ser superado pelas técnicas meta-heurísticas AG e DE.

Em uma comparação entre a sintonia fornecida via AG e DE, é evidente (ao menos em termos da ISE) que o primeiro forneceu uma melhor resposta do que a segunda técnica. Em termos de tempo necessário para atingir o regime permanente a sintonia via 
AG e a sintonia via DE tiveram tempos praticamente iguais, cerca de $3 \mathrm{~s}$ como pode ser constatado na Fig. 3 e da Fig. 4. De um modo geral, e do ponto de vista gráfico, é possível afirmar que a sintonia de ambos os métodos meta-heurísticos são equivalentes

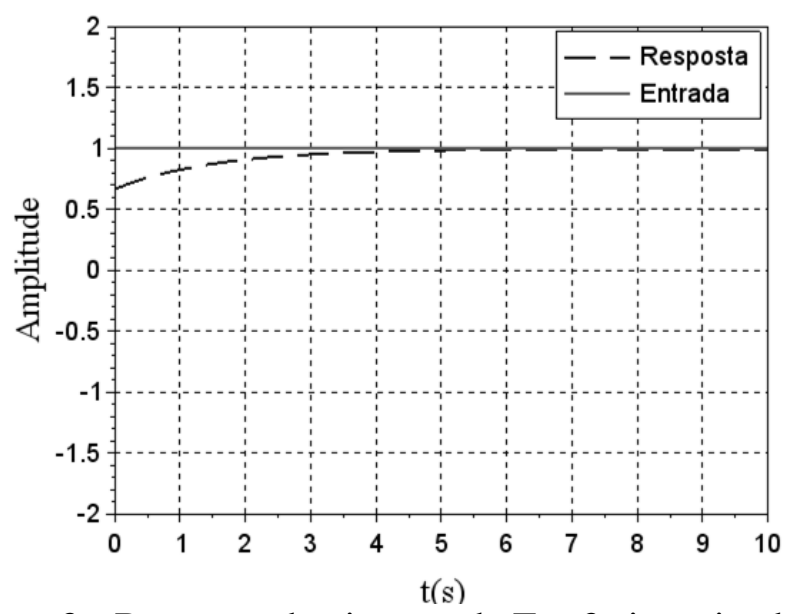

Figura 3 - Resposta do sistema da Eq. 3 sintonizado via DE.

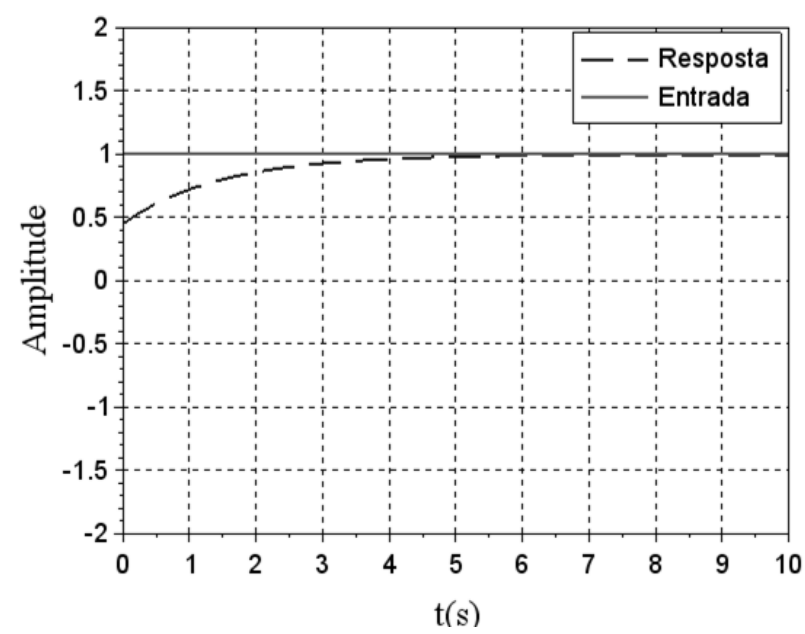

Figura 4 - Resposta do sistema da Eq. 3 sintonizado via AG.

\section{CONCLUSÃO}

Ao final deste trabalho é possível fazer algumas inferências. A primeira delas é a de que os métodos meta-heurísticos AG e DE são capazes de fornecer uma melhor sintonia do que o método ZN. Fato que é confirmado por uma comparação gráfica das respostas e pelo critério de desempenho ISE. Outra conclusão é a de que, dentro das condições consideradas neste trabalho, a sintonia via AG é capaz de fornecer uma sintonia relativamente melhor, em termos da ISE, em relação a sintonia via DE.

\section{REFERÊNCIAS}

1. H. S. Sánches; R. Vilanova; O. Arrieta. Implementación de controladores PID: Equivalencia y Optimalidad, in: XXXV Jornadas de Automática, Valencia, 2014. 2. ENTERPRISES, Scilab. About Scilab. Disponível em: < http://www.scilab.org/scilab/about>. Acesso em: 10 jan. 2017.

3. KOEPSEL, Roberto Navarro. Módulo experimental para sintonia de sistema de controle antecipatório. 60 f. Desertação (Mestrado em Engenharia Química). Programa de Pós-Graduação em Engenharia Química, Universidade Estadual de Maringá. Maringá, 2018. 